\title{
A Platform for Parameter Unlimited Molecular Geometry Imaging Obviously Enabling Life Saving Measures in ALS
}

\author{
Walter Schubert ${ }^{1,2}$ \\ ${ }^{1}$ Molecular Pattern Recognition Research Group, Medical Faculty, Otto von Guericke University, Magdeburg, Germany \\ ${ }^{2}$ Human Toponome Project, TNL, Munich, Germany \\ Email: walter.schubert@med.ovgu.de
}

How to cite this paper: Schubert, W. (2018) A Platform for Parameter Unlimited Molecular Geometry Imaging Obviously Enabling Life Saving Measures in ALS. Advances in Pure Mathematics, 8, 321-334. https://doi.org/10.4236/apm.2018.83017

Received: February 15, 2018

Accepted: March 24, 2018

Published: March 27, 2018

Copyright $\odot 2018$ by author and Scientific Research Publishing Inc. This work is licensed under the Creative Commons Attribution International License (CC BY 4.0).

http://creativecommons.org/licenses/by/4.0/

\section{(c) (i) Open Access}

\begin{abstract}
This article deals with a molecular geometry imaging platform capable of mapping the spatial protein-colocalisation and anti-colocalisation code of large molecular systems at a time. The platform called molecular unlimited systems imaging cycler (MUSIC) robotics was applied to amyotrophic lateral sclerosis (ALS). The detection of ALS specific cells with a corresponding multimolecular geometry in the blood led to therapeutic depletion of these cells and to recovery of the treated patient, obviously because this therapy interferes with pathogenic invasion of these cells into the central nervous system, where they axotomize motor axons. Large scale geometry MUSIC robotics imaging of up to $4.5 \times 10^{481}$ power of combinatorial molecular resolution is key to detect these cells and to control depletion therapy for clinical success. These data and new possibilities may argue for clinical application and for a systematic research in the field of molecular geometry of diseases to discover new mathematically defined insight.
\end{abstract}

\section{Keywords}

Geometry, Toponome, ALS, Motor Neuron Disease, Imaging, Toponome-Therapy

\section{Introduction}

All life processes are spatial. Therefore, the molecular networks controlling life processes must also follow spatial laws. In order to understand these laws, methods must be developed that allow these networks to be imaged in a hypothe- 
sis-free manner, for an unlimited, or at least extremely large number of defined molecules and to directly distinguish the disease specific from the non specific networks in the morphologically intact tissue, optionally in real time. The development of the molecular unlimited systems imaging cycler (MUSIC) robotics method represents a fundamental technique that allows us to spatially map the so-called toponome, the spatial protein network, as a geometric data set with hundreds or thousands of interlinked combinatorial molecular clusters, to detect the resulting so called lead proteins or whole cells controlling these networks [1] [2]. These steps have proven to be fundamental when it comes to understanding their laws and eliminating disease-specific, so-called robustness networks, in a targeted therapeutic way [3], as shown here for ALS.

The question of whether these natural processes follow exact combination rules of proteins in health and disease has been answered in recent years by the imaging cycler robotics technology in healthy and diseased human tissues [1] [3] [4] [5] [6] [7]. According to this, protein systems in tissues can be understood as a system that follows a kind of spatial protein association, tissue-bound "grammar" and a protein association "vocabulary". To decode this system is therefore mandatory when it comes to understand diseases because of the following reasons: with only a limited number of rules for the spatial tissue association of e.g. proteins, a quasi-unlimited number of spatial "texts" (diseases) with different contents (toponomes) can be expressed in tissues. These "texts" can, for example, be understood as spatial blueprints for disease, which can be decoded directly by means of imaging cycler robotics as a toponomical data set [7] [8] [9]. These spatial blueprints, in turn, typically include so-called emergent systems [10], where emergence is understood as the process of complex pattern formation from more basic constituent parts [10]: large combinatorial molecular structures acquire, e.g. through the differential interplay of the numerous different protein classes, new properties in health and disease [10].

To elucidate these characteristics as specific features of diseases appears to be the "conditio sine qua non" for the development of efficient treatments of chronic diseases, such as cancer or amyotrophic lateral sclerosis (ALS) [10]. This is supported by recent clinical success [11] based on toponome predicions [7] concerning the lead protein function of aminopeptidase $\mathrm{N}$ in cancer. So it's bound to be very erroneous to assume that from lists of extracted proteins (from tissue) disease-specific information can be gained. On the opposite: there must be a very specific spatial combinatorial order of proteins: this view is further supported by the fact that proteins in the Golgi apparatus of the cell are immediately sorted into specific, highly non random contexts with other proteins in vesicles which are then transported to their destination in the same cell and so on. Hence, there are plenty of biologically and anatomically motivated reasons speaking for a systematic decoding of large molecular systems in their natural structurally defined environment. Here the fundamentals of MUSIC robotics geometry mapping and the case of ALS are described as an example. 


\section{MUSIC Robotics Platform Bypassing the Spectral Resolution Limit}

The detection of several different proteins in tissue sections or single cells, e.g. the detection of 4 different proteins at a time, is possible in optical routine microscopy with the aid of fluorescence microscopy. But fluorochrome-specific wavelengths must be selected with the aid of filters, which limits the colocalization of different protein classes down to only 4 to 5 within the limited spectrum of visible light ranging from 300 to $700 \mathrm{~nm}$ wavelengths: too few separable parameters to determine the high-dimensional networks of proteins or other molecular classes to analyse and understand their higher order combinatorial laws. However, due to the so-called sunlight hypothesis set up by the author of the present article in 1987 and described in [10], these limitations can be overcome or bypassed by using only one wavelength trace with sunlight-like soft scattered light to sequentially map many different molecules (100 or 1000 or more) with only one fluorochrome, if the fluorochrome is gently bleached before the next round for the next molecule is run. This so called sunlight hypothesis was experimentally verified in a two-page paper [12] and henceforth used for the development of an automated platform: the molecular unlimited systems imaging cycler robotics technology.

\section{Direct Detection of Emergent Molecular Systems in Human Tissue}

Two methods for the visualization of emergent molecular systems have been developed: the method of threshold-based analysis of molecular networks [7] [9] [12] [13] and the so-called similarity mapping method [14]. Both methods allow for analysis of high-dimensional molecular networks directly in the tissue on the basis of visualizing and quantifying a relative molecular stoichiometry of the different molecules (Figure 1). The threshold-based method reveals so-called lead proteins that control large molecular networks (Figure 1, top middle) [7] [8] (see also note added in proof). This provided evidence that the blocking of such lead proteins leads to a dramatic effect, by decomposing entire networks of proteins spatially [10]. For example, inhibition of the enzyme aminopeptidase N (APN), which controls a complete molecular network as a lead protein in rhabdomyosarcoma cells, results in the complete collapse of the molecular network, which normally enables cells to reach the elongated migration status [7]. Hence, in the meantime, this blocking method for this enzyme in cancers has been in positive validation as a therapeutic target in numerous clinical trials [11]. These data show that the MUSIC robotics method for detecting such relationships in disease-specific emergent systems is, for example, aimed at developing efficient therapeutic methods.

The direct detection of emergent molecular systems in one and the same type of tissue shows the MUSIC robotics mapping of 100 different molecules in one tissue section of the human skin: (Figure 1, Figure 2) [4]. Two methods of protein cocluster analyses are illustrated: Figure 1, top line, shows the MUSIC robotics 
a
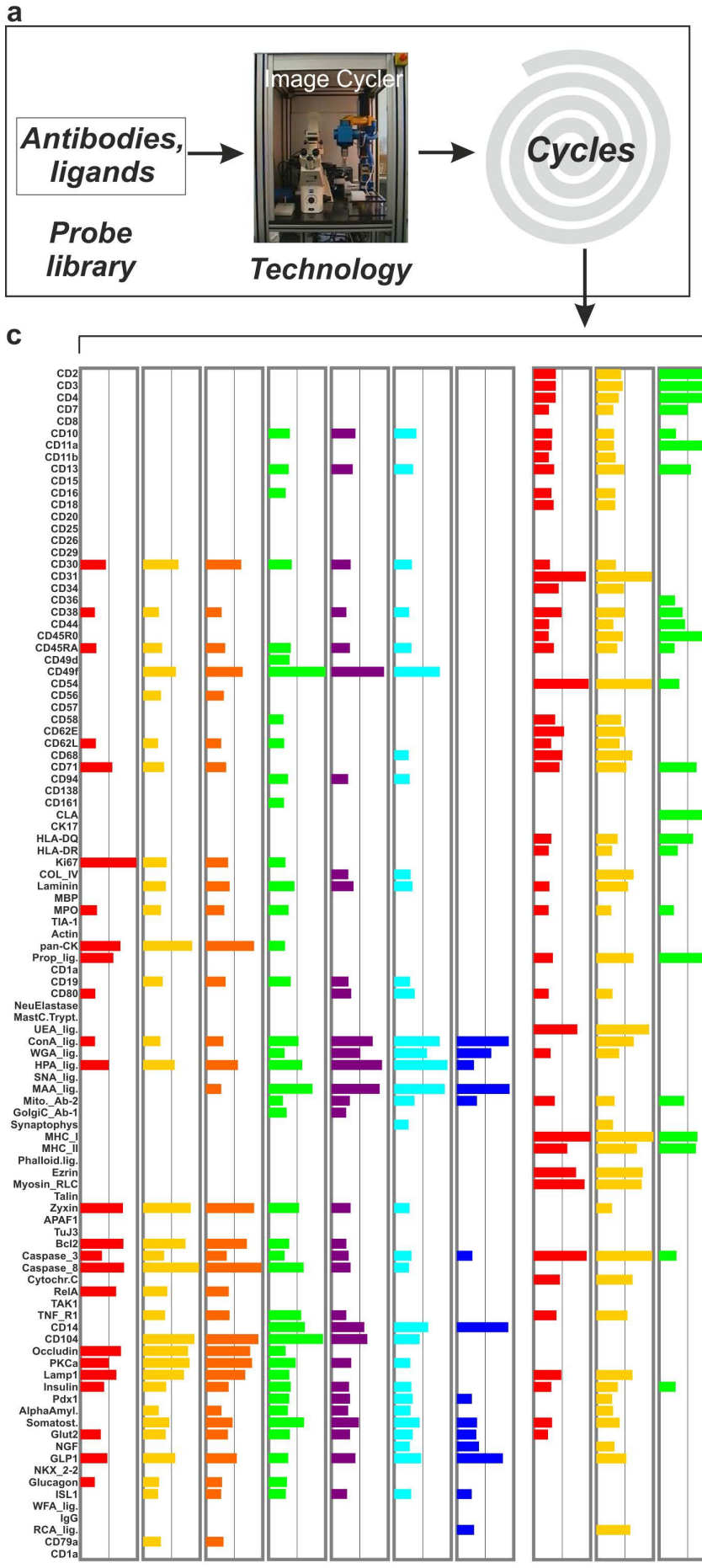

b
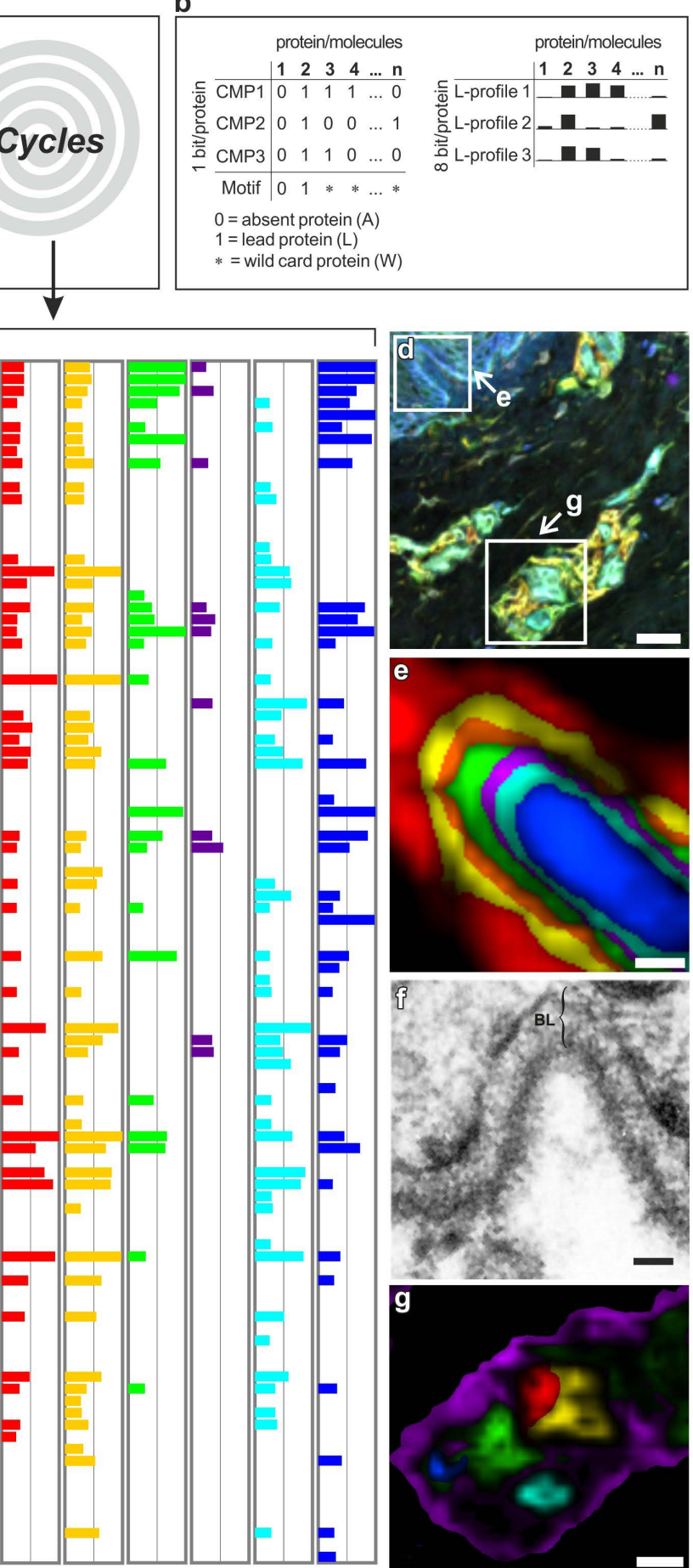

Figure 1. Principles of detecting distinct molecular geometries by real time protein profiling in situ. The topline of the figure illustrates the MUSIC technology platform running automated cycles of incubation, imaging and bleaching to reveal large scale molecular profiling and its decoding by a threshold based method ( 0.1 code) (ref 9 ) or by similarity mapping in real time (shown on the right in direct comparison with the threshold based method, ref 14). Example of similarity mapping in human skin tissue section was used to show the profiles indicated in the boxed areas in (d) and (g). Similarity mapping of the area of (d) reveals 6 distinct layers of the basal lamina (different colors show the profiles with their structure in (e)). This whole dimension is $120 \mathrm{~nm}$ in electron microscopy analyses (f). Another area of the skin (boxed area g) shows several other protein profiles which are specifically expressed in this area. Together, 13 distinct 100-component profiles are detected as specific features of substructures within this human tissue. 

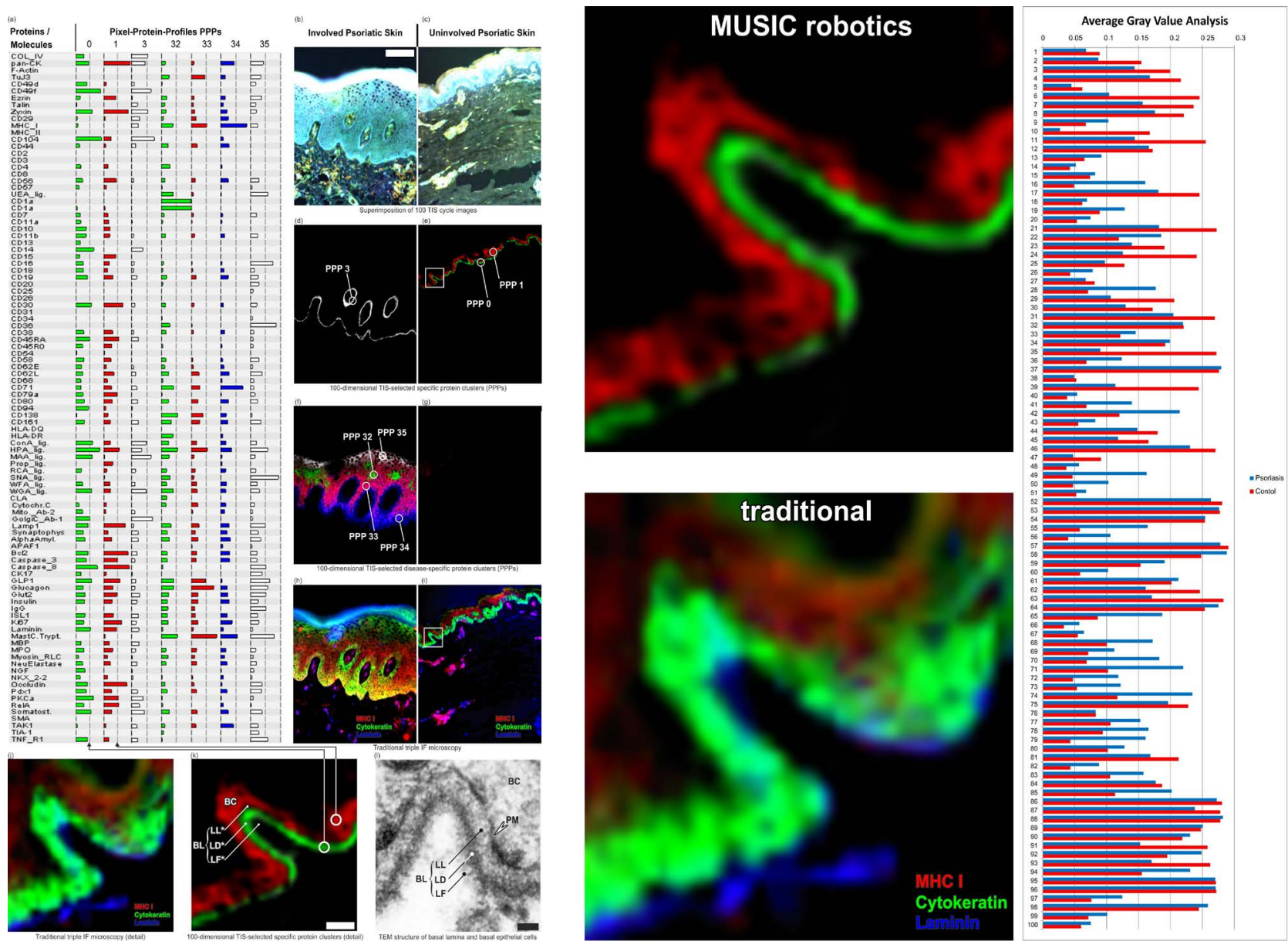

Figure 2. Principle of proving emergent structures in tissue. Direct comparisons in one and the same human tissue section of diseased skin (left vertical series of images) and normal skin (parallel arrangement of images) reveals highly specific pixel protein profiles (PPP) in a disease specific way (colored PPP on the left). Note: only these large scale PPP reveal very sharp distinct structures (image top right), while simple colocalization of 3 of its components (lower image on the right) reveals the natural localisation of the corresponding molecules. In the same set up the same molecules however contribute to the visualisation of three separated substructures (upper image on right). This shows that emergent structures cannot be identified by traditional techniques and not by large scale profiling without anatomical resolution (on the right), but can be seen only, if a very large number of molecular components is visualized simultaneously at concrete anatomical sites. On the right: non antomical (average) protein profiling.

principle schematically, and the threshold based and non threshold based protein profiling method. It can be seen in the detail enlargement of Figure 1 (right vertical panel of images), that the MUSIC robotics analysis reveals 6 layers of the basal lamina (Figure 1(e)) (image on right, in the middle of the panel), which by electron microscopic analysis (Figure 1(f)), reveals only 120 nanometers width. Hence MUSIC robotics reveals 6 functionally distinguishable layers with a total width of the EM structure of $120 \mathrm{~nm}$, now enabling us to differentiate this structure with an optical microscopic setup. Furthering this observation: The colocalization of only 3 molecules (Figure 2, right hand side, bottom) out of the 100 co-mapped proteins, which are also part of the overall profile (Figure 2, left hand side), shows at the same basal lamina site the real localization of these molecules, which show a much broader presence than expected from the 100-profile 
(Figure 2, left hand side). This is not a contradiction, on the contrary: Only the complete structural feature of the system comprising 100 distinct proteins visualized by the similarity approach (14) (Figure 2, left hand side) shows emergence, that is, the structure to which the 3 colocalized proteins contribute as part of the whole, while colocalization of only 3 proteins does not result in any emergence, as revealed by using the same optical setup in the same tissue for these correlations. With such comparisons in one and the same data set, one can directly prove emergence by means of MUSIC robotics, and thus apply this disease specificity as a starting point for targeted therapeutic procedures.

\section{Emergent Molecular System in ALS}

The sporadic, non-genetic form of amyotrophic lateral sclerosis (ALS) affects approximately $90 \%$ of ALS patients. The cause of the disease is unknown. Its clinical course is characterized by unstoppable progressive motor disabilities leading to death after a few years in most of the cases, or even earlier. An efficient therapy is not known. More than 50 clinical trial, all based on hypotheses, have failed [15].

The search for emergent molecular systems in the sense of a disease-specific molecular geometry in ALS by using MUSIC robotics appeared promising in view of the methods depth of the content of information in cells and tissues. From the years of systematic development work of MUSIC robotics applications, an informative labeling library was selected [12] [13] and applied by MUSIC robotics to the analysis of mononuclear blood cells [3] and post-mortem tissue from ALS cases in the year 2003 in our lab (project BainNet approved for WS).

As shown in Figure 3, inside the vascular lumen of a postcapillary venule, hence inside the blood stream in ALS, a cell form can be detected by MUSIC robotics, but not by traditional methods, that permeates the vascular endothelium of a postcapillary venule in the area of the lateral tractus pyramidalis (corticospinalis lateralis) in the spinal cord, one of the tract systems of voluntary motor function. This condition is therefore to be interpreted as a post-rolling condition of this cell that enables the cell to polarize and initiate the transendothelial invasion process, hence breaking the blood brain barrier. After invasion, such $\mathrm{CD} 8 / \mathrm{CD} 3$ positive cells are found in the area of the pyramidal tract (Figure $4(\mathrm{~g})$ ). High dimensional molecular geometry imaging of the blue/red structure of Figure 4(g), reveals a T cell similar cell, that extends a CD8 and CD3 expressing cell extension towards the axon of a pyramidal neuron (Figure 4(h), Figure 4(i), asterisk) indicating that this process drives an axotomy or at least axon compression of an intact motor axon, possibly in the area of a Renvier node, while myelin sheaths appear to be intact (Figure 4(k), white structure). This pathology was previously unknown because of a lack of combinatorial molecular visualization methods with emergence resolution, so that the pathogenesis of ALS was not resolvable at high functional resolution [3]. The conservative colocalisation of the two markers CD8 and CD3 (Figure $4(\mathrm{~g})$ ) is, analogous to 

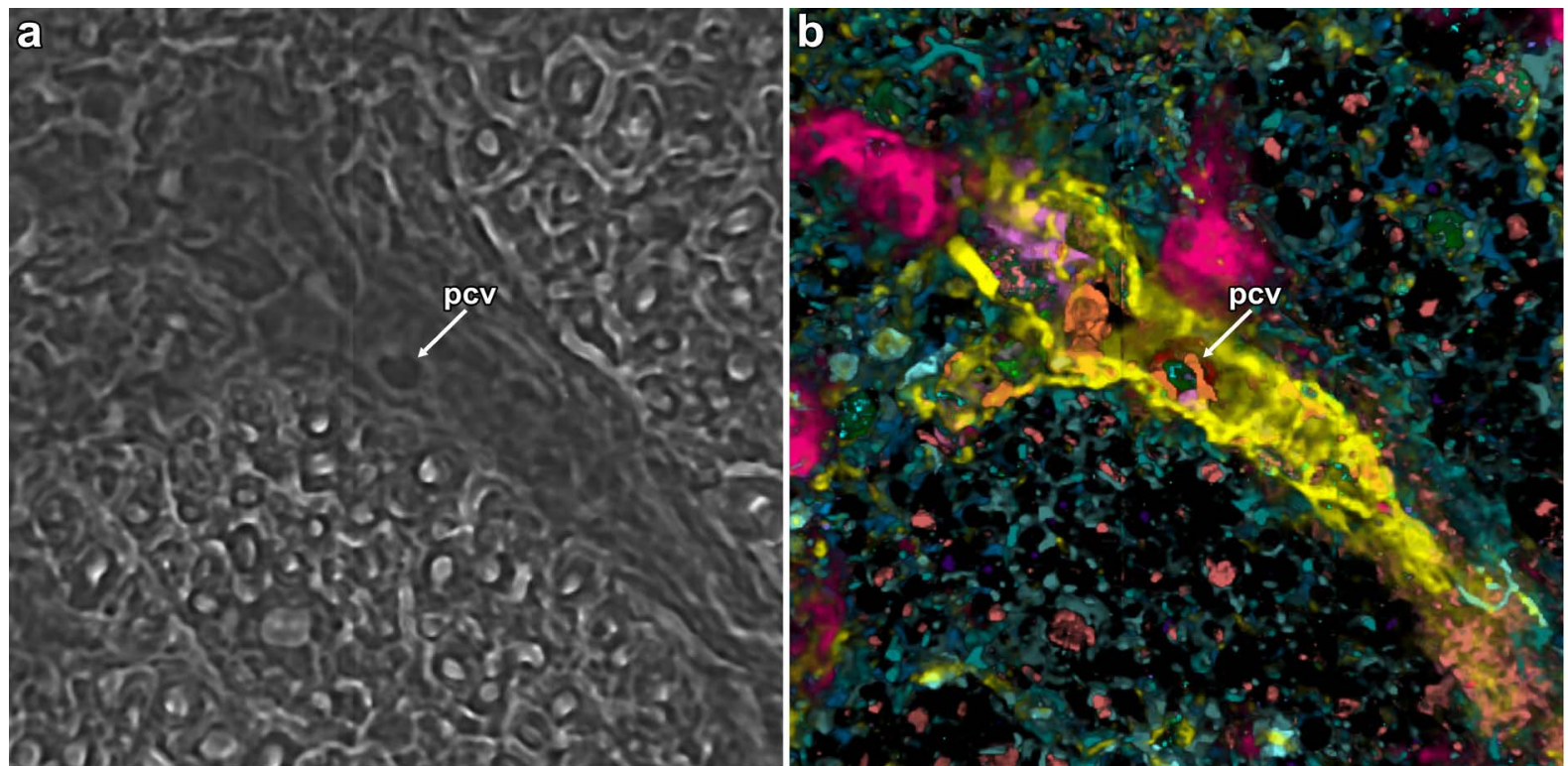

Figure 3. Cell inside the blood stream prepared to crossing the blood brain barrier in ALS. (a) post mortem tissue showing a mononuclear cell inside a post capillary venule (Phase contrast, arrow); (b) This cell (arrow in b) expresses a large MUSIC robotics mapped molecular profile, part of which is shown in colors in direct comparison with the cell feature in (a, arrow). The features signify the cell prepared to cross the blood brain barrier.
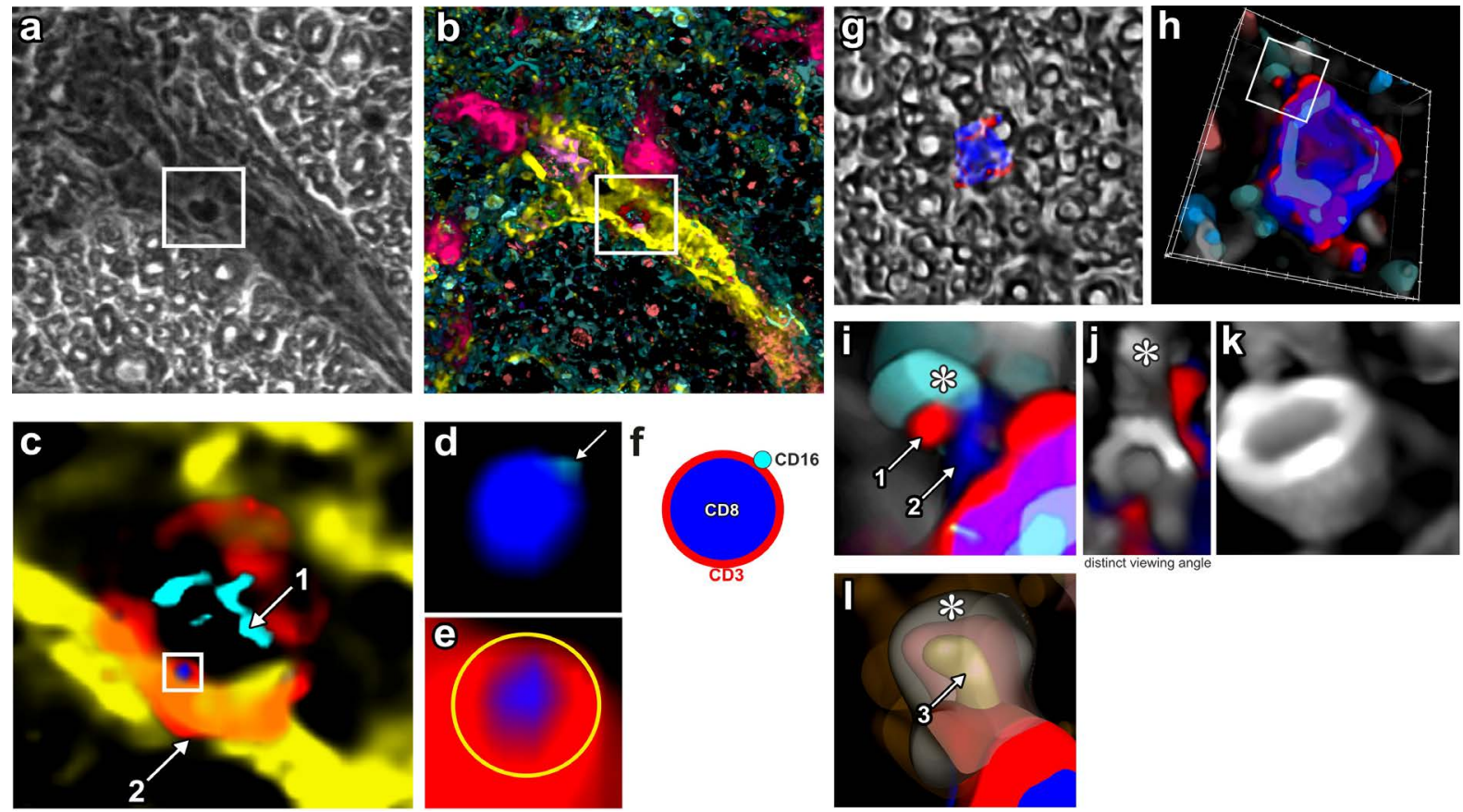

Figure 4. Synopsis of the molecular geometry of ALS in post mortem tissue. (a) to (k) is described in the text. In addition, the fact that the cell (c) inside the blood vessel is crossing the endothelial barrier (yellow) is given by the fact that its cell membrane (red) overlaps with the endothelial cell profile (yellow) and has already reached the nervous tissue as indicated by the arrow 2 in the image (c). During this transendothelial migration the cell extrudes vesicles containing CD16 and CD8, because these two lead proteins are not needed in this cell state any more: they were essential part of the cell state of rolling having preceded the cell state of transmigration. (g) After invasion the CD8 CD 3 positive cell is found in the parenchyma between myelinated motor nerves ( $\mathrm{g}$, blue and red dots). If however MUSIC robotics is applied, these structures resolve as a cell that compresses a motor axon ( $h$, boxed area) and (i) in higher magnification. $(j)$, (k) this process leaves the myelin sheaths intact. 
the triple labeling shown in Figure 2, not recognizable as an emergent structure: if, however, the entire MUSIC robotics data set is used for this cell, this cell exhibits the following mechanism: it displaces a motor axon (Figure 4(i)). Such processes do not exist in the healthy motor system of the pyramidal tract. None of the described cell invasions were found in the postmortem control study with tissue from persons having died suddenly because of heart disease. It can therefore be assumed that cell invasion and consecutive axotomy by the described cell, henceforth called axotomy-competent-cell (ACC), are specific for sporadic ALS. Progressive motor neuron phenomena of the disease, such as progressive paralysis, breathing insufficiency, and bulbar symptoms are likely to be caused or at least driven by the described cell type. This cell type is therefore referred to as axotomy-competent cell (ACC). The number of ACC per litre blood correlates with the rate of progression of the disease substantiating its proximal pathogenic role.

A MUSIC robotics magnification of an ACC in its active function of axotomizing a motor axon (Figure 5(b)) shows that the CD3 and the CD8 receptors are polarized in an aberrant distance of $855 \mathrm{~nm}$ to each other (Figure 5(b)), while the requested distance for an antigen recognition-driven transduction of an activating signal into the cell would require a distance less than $1 \mathrm{~nm}$ as shown schematically in Figure 5(a). Hence it is unlikely that this cell is able to lyse the axon by an antigen-driven mechanism. This indeed is in strong keeping with the fact that the axoplasm (Figure 5(b), asterisk) is homogeneous instead of being lysed. Hence, the axotomy is resulting from a displacement process exerted by the ACC, actually an axon-compression. This is in keeping with earlier observations having been made by an initial application of MUSIC robotics: the whole postmitotic system, including the endomysial tube of skeletal muscle fibres is protected from being cytotoxically attacked by invasive $\mathrm{T}$ cells, because such cells do not activate early response gene products [13] [16].

\section{Therapeutic Depletion of the ACC}

It seems likely that, in the context of postmortem data (Figures 3-5) and recent evidence (3) ACC should be therapeutically destroyed or depleted as early as possible in ALS patients. Since the molecular geometry, respectively the toponome of the ACC in the blood is given [3] (Figure 6(a)), it is conclusive to find these cells in the blood also in a simple blood cell test, under the prerequisite that MUSIC robotics is applied. This was shown to be the case recently [3], and high resolution protein profiling of such ACC (Figure 6(b)) shows directly that the corresponding spatial molecular profile of a patient diagnosed with signs of ALS (neurplogically diagnosed) largely corresponds to the ACC invading the pyramidal tract (compare Figure 6(a) with Figure 6(b)) both expressing a very similar if not identical molecular profile with $\mathrm{CD} 8 / \mathrm{CD} 16$ lead proteins (Figure 6).

Therapeutic depletion of this ACC cell type (Figure 6(b)) in a patient with diagnosed ALS/motor neuron disease and follow-up over approx one year 

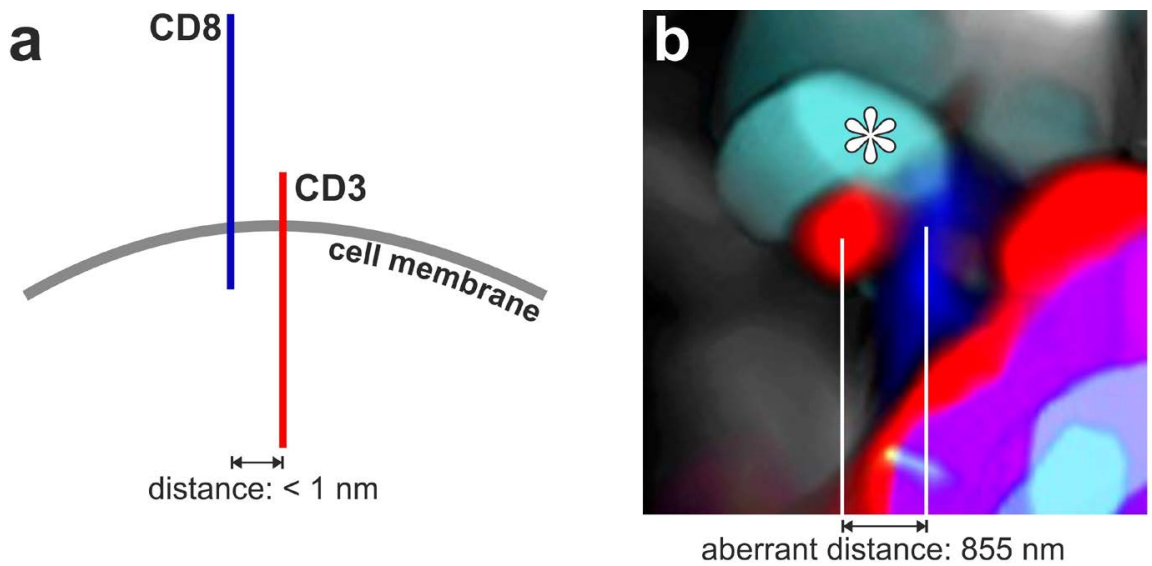

Figure 5. The pathogenic process of axon compression by an invasive cell. (a) Schematic illustration showing that a $\mathrm{T}$ cell needs a minimum distance of the CD8 and CD3 receptor to be able to transmit a signal across the cell surface membrane. (b) The invasive cell compresses the axon by extending separated CD 3 positive (red) and CD 8 positive (blue) cell extensions toward the motor axon (asterisk). (b) The invasive axon compressing cell displays CD3 (red) and CD8 (blue) in a distance of $855 \mathrm{~nm}$, which is not compatible with an efficient antigen-driven signal transduction.

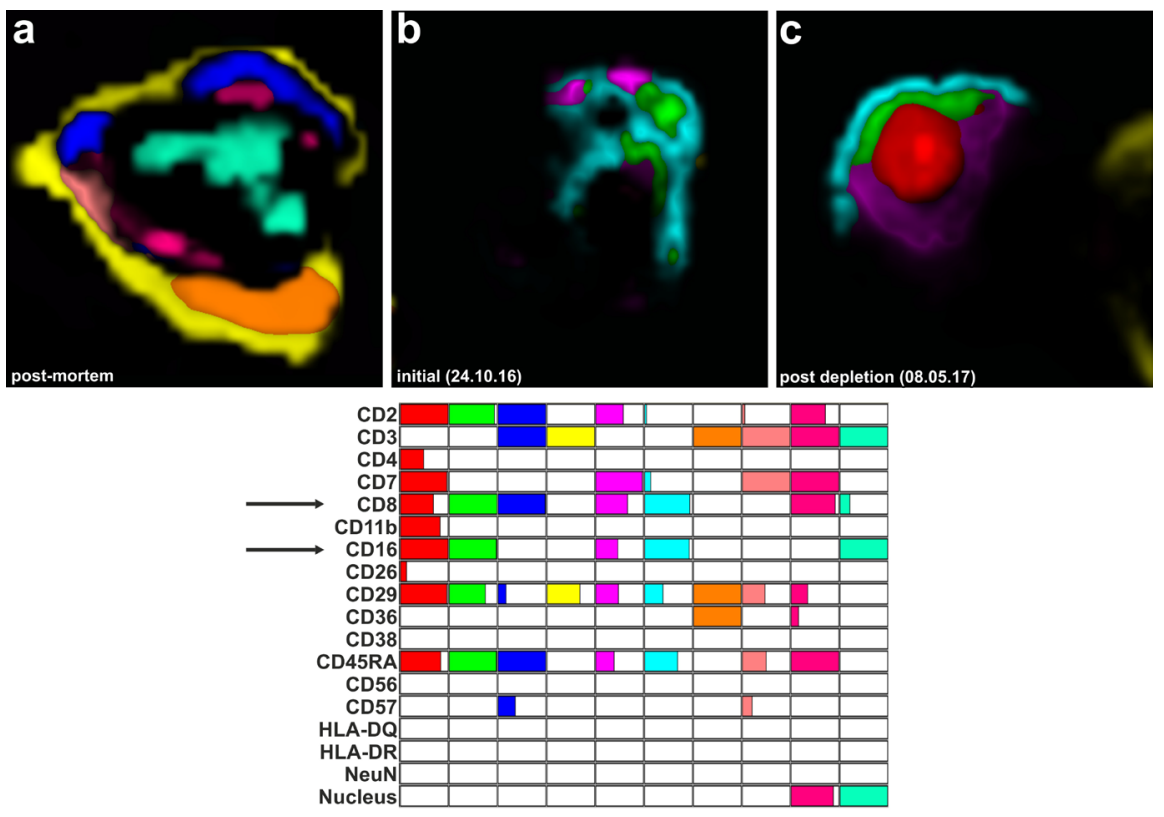

Figure 6. Detection of axotomy competent cells in the blood. (a) Original geometry of the invasive axotomy competent cell inside the blood vessel of the pyramidal tract as detected in a post mortem study. The endothelium of this vessel is colored in yellow, which expresses the corresponding molecular profile as indicated in the profile map below the images. (b) The colors in this image correspond to the invasive cell (master cell) in (a), as revealed by MUSIC robotic profiles below the images. Arrows indicate presence of so called lead proteins. (c) A therapeutic method leads to a damage of this cell type that becomes dysfunctional. (c) Profiles below the images indicate in different colors the protein profiles that are present in these cells $(a-c)$. Because the profile in (b) corresponds to the profile of the invasive cell in (a) inside the blood vessel, the cell of (b) is identified as an axotomy competent cell (ACC), which is the indication for its therapeutic alteration shown in (c). 
resulted in the disappearance of neurological signs of the disease and subjective well-being (clinical details to be published elsewhere).

Figure 7 displays the number of ACC in blood over time in a patient treated by ACC depletion. This procedure correlated with the neurological findings briefly described above, showing no pathological spontaneous activity in electromyogram anymore. Another 4 patients with motor deficits in advanced ALS showed the same ACC cells as documented in this case reported here for one patient. Depletion of these ACC was possible also in these more progressed stages of ALS (to be reported in detailed clinical form).

The reason for the therapeutic effect in the one case reported here appears to be that the invasion specificity of the ACC expressing a specific combinatorial molecular structure at the cell surface for the capillary endothelium inside the pyramidal tract (Figure 8) is removed by depletion (Figure 7) or by damaging the functional cell structure of the ACC (Figure 6(c)).

\section{Discussion and Conclusion}

The results described here were found by MUSIC robotics microscopy, which was previously used with a combinatorial molecular resolution ranging from $4.31 \times 10^{86}$ to $4.4 \times 10^{481}$. The resulting detected high-dimensional geometries in human tissue reveal disease-specific emergence and disease-specific cells in ALS, whose role in this disease can be fully decrypted: the cells whose axotomy competence could be well understood by post-mortem analysis using MUSIC robotics. It led to the conclusion that these cells appear to be causative for the pathogenesis of sporadic ALS: 1) ALS specific cells in the blood are broadly consistent with the CNS-invasive cell type, which compresses motor axons in ALS

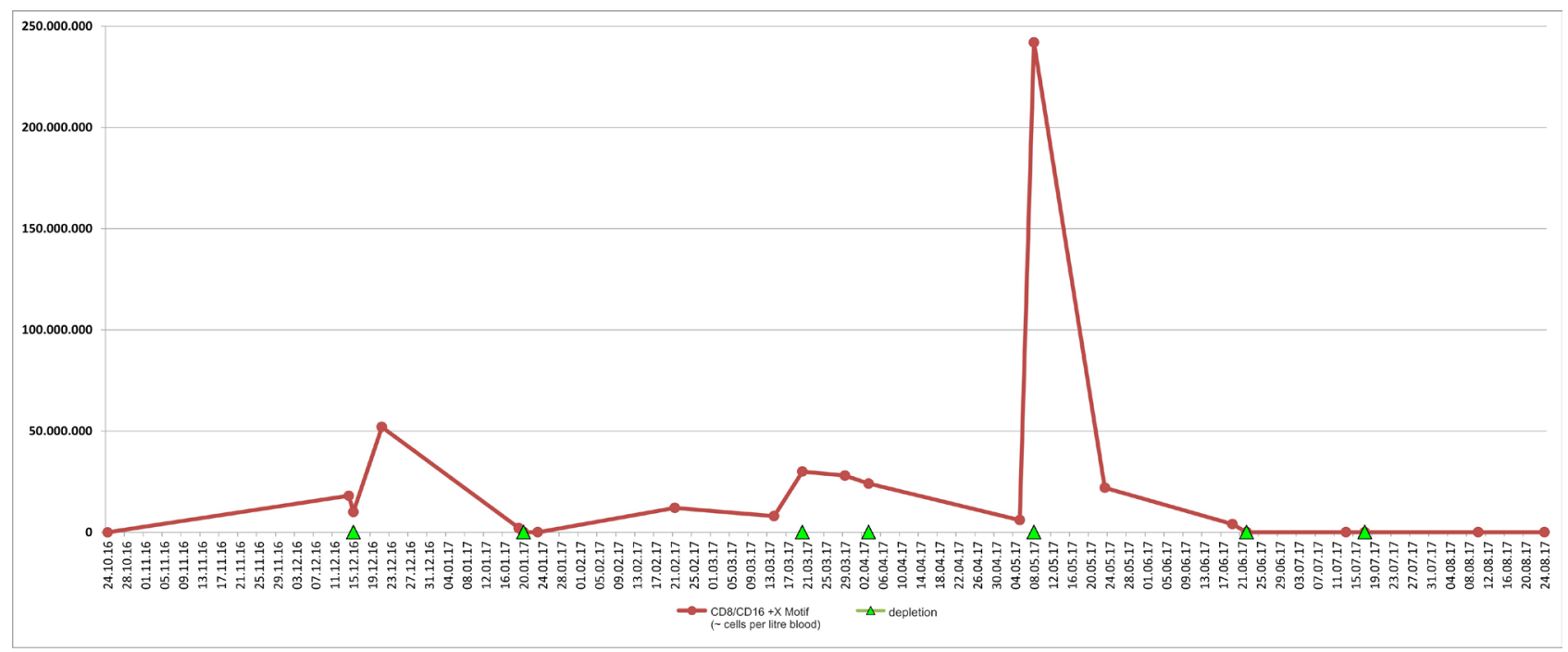

Figure 7. Illustration of the effect of ACC depletion in a patient. The red line plots the number of ACC (y axis, number of ACC per litre blood) over time ( $\mathrm{x}$ axis), as detected and controlled by MUSIC robotics. The depletion therapy shows that over long periods of time following depletion therapy the ACC are not detectable any more. On the other hand sudden "eruptions" of ACC numbers can be detected by MUSIC robotics (peak) providing the possibility to enhance the depletion therapy in order to counteract this process. 


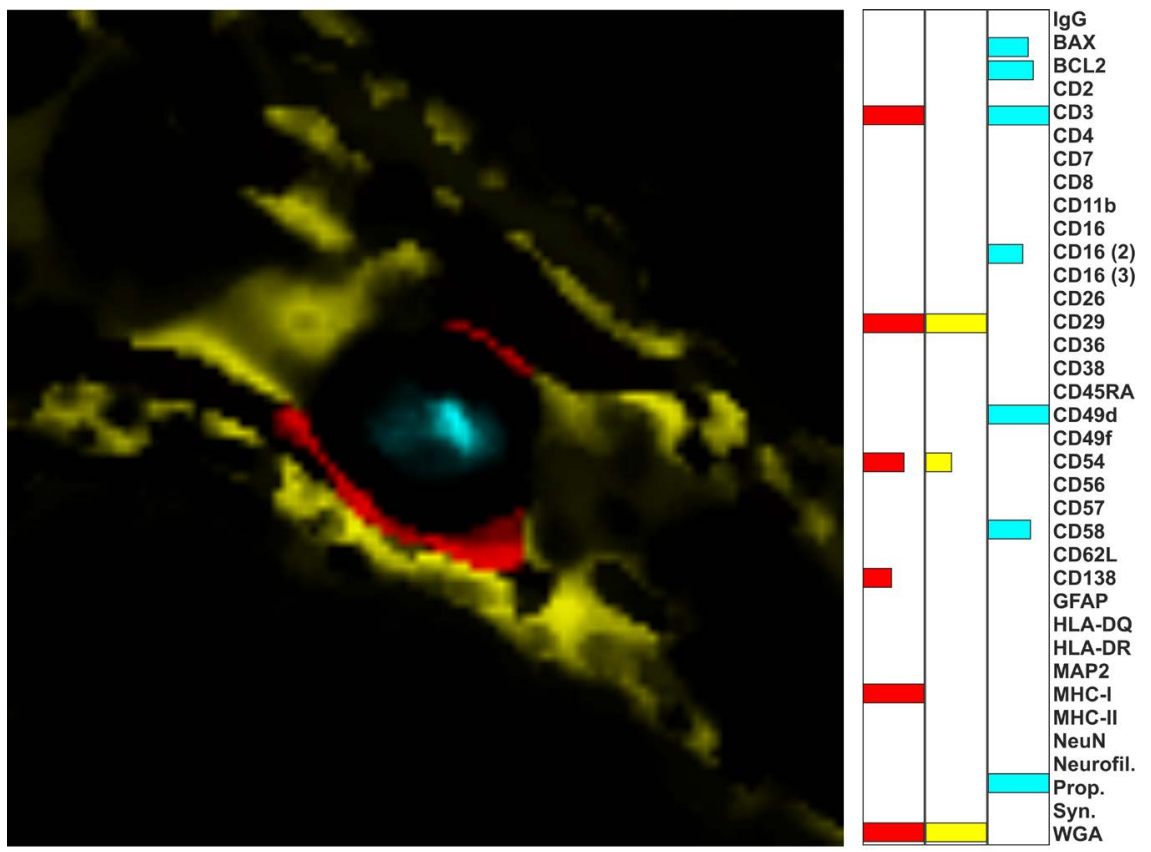

Figure 8. Optical MUSIC robotics section across the invasive ACC. The yellow structure belongs to the endothelium of the post capillary venule (see aforementioned figures), and red structure belongs to the cell surface membrane of the blood borne ACC. The blue structure belongs to the internalized CD16 coreceptor of the combinatorial molecular cell surface structure that the ACC has needed for endothelium recognition in a slightly earlier stage. Indeed the shown ACC surface protein combinations (red) and anti combiantions (white spaces ) may represnt an ALS specific cell surface code (red) that recognizes the endothelial address code (yellow). Indeed this is substantiated by the fact that this profile was never found in healthy individuals or patients with other diseases (to be published).

and thereby significantly affects them;2) if these cells are specifically depleted from the blood circulation, then there is a significant regression of the disease phenomena accompanied by physical well-being (neurologically documented). This association and the fact that they also occur in other ALS patients with the sporadic form of the disease, where the number of these cells per litre of blood correlates with the course rate of the disease, support the conclusion that these cells are causal for the sporadic ALS: Fifty million cells per one litre blood correspond to a threefold slower speed rate of ALS than 150 million cells. Since the patient diagnosed here and treated with cell depletion appears to be clinically healthy again, one can complete the following: the coupling of cycles "MUSIC robotics detection of the cells-then depletion of the cells-control of the measure using MUSIC robotics, etc." seems to be a life-saving measure, if one considers that the disease is always strictly progressive and leads unavoidably to death. The data are compatible with the observation of other investigators, who showed that the molecule TDP-43 in neurons increases and accumulates in ALS [17]. Axotomy is the natural cause of neuronal TDP-43 accumulation (17). However, it cannot be ruled out that sporadic ALS might have several other forms of pathogenesis. However, in the case of sporadic ALS, when ACC is detected, ACC depletion is an indication as a life-saving measure, given the data 
presented here. The corresponding medical indication should be made as early as possible to prevent irreversible damage of the motor system. The MUSIC robotics technology and corresponding protocols have been described in detail [18]-[24]. The experiences reported here might stimulate a systematic mathematical approach to the molecular geometry of emergence mechanisms in diseases, which cannot be revealed by large scale expression profiling without anatomical resolution, as illustrated in Figure 2, on the right. Translation of the method described here into broad therapeutic application for ALS patients is in preparation.

\section{Acknowledgements}

Supported by the Deutsche Forschungsgemeinschaft (DFG Schu627/10-1), BMBF (grants CELLECT, NBL3, NGFN2, and NGFNplus), DFG-Innovationskolleg (INK15), EU IMAGINT (Health-F5-2011-259881), Human Toponome Project (http://www.toposnomos.com/huto/index.html), and the Berliner Krebsgesellschaft (WAFF2000824), the Klaus Tschira foundation (KTS), and the face ALS foundation.

The continuous technology development, based on the basic principle [12], was done by two teams over time: Team 1: Christoph Rethfeld, Uwe Setzer, Marcus Bode, Lars Philipsen, Manuela Friedenberger; Team 2: Andreas Krusche, Reyk Hillert, Anne Gieseler. Substantial support was provided by the Max-Planck-Chinese Academy of Sciences (MPG-CAS) partner institute for computational biology, Shanghai, China (international faculty), and the Freiburg university, Germany, for WS as professor of toponomics.

\section{References}

[1] Schubert, W. (2010) On the Origin of Cell Function Encoded in the Toponome. Journal of Biotechnology, 149, 252-259. https://doi.org/10.1016/j.jbiotec.2010.03.009

[2] Schubert, W. (2013) Toponomics. In: Dubitzky, W., Wolkenhauer, O., Yokota, H. and Cho, K.-H., Eds., Encyclopedia of Systems Biology, Springer, New York, Heidelberg, 1291-2212.

[3] Schubert, W. (2015) Advances in Toponomics Drug Discovery: Imaging Cycler Microscopy Correctly Predicts a Therapy Method of Amyotrophic Lateral Sclerosis. Cytometry Part A, 87, 696-703. https://doi.org/10.1002/cyto.a.22671

[4] Schubert, W., Gieseler, A., Krusche, A., Serocka, P. and Hillert, R. (2012) Next-Generation Biomarkers Based on 100-Parameter Functional Super-Resolution Microscopy TIS. New Biotechnology, 29, 599-610. https://doi.org/10.1016/j.nbt.2011.12.004

[5] Ostalecki, C., Lee, J.H., Dindorf, J., Collenburg, L., Schierer, S., Simon, B., Schliep, S., Kremmer, E., Schuler, G. and Baur, A.S. (2017) Multiepitope Tissue Analysis Reveals SPPL3-Mediated ADAM10 Activation as a Key Step in the Transformation of Melanocytes. Science Signaling, 10, eaai8288. https://doi.org/10.1126/scisignal.aai8288

[6] Abbott, A. (2006) Mapping Togetherness (Research Highlight). Nature, 443, 609. (Referring to Schubert et al. 2006) 
[7] Schubert, W., Bonnekoh, B., Pommer, A.J., Philipsen, L., Boeckelmann, R., Malykh, Y., Gollnick, H., Friedenberger, M., Bode, M. and Dress, A.W. (2006) Analyzing Proteome Topology and Function by Automated Multidimensional Fluorescence Microscopy. Nature Biotechnology, 24, 1270-1278.

[8] Schubert, W. (2006) Exploring Molecular Networks Directly in the Cell. Cytometry $A, 69,109-112$. https://doi.org/10.1002/cyto.a.20234

[9] Schubert, W. (2007) A Three Symbol Code for Organized Proteomes Based on Cyclical Imaging of Protein Locations. Cytometry A, 71, 352-360. https://doi.org/10.1002/cyto.a.20281

[10] Schubert, W. (2014) Systematic, Spatial Imaging of Large Multimolecular Assemblies and the Emerging Principles of Supramolecular Order in Biological Systems. Journal of Molecular Recognition, 27, 3-18. https://doi.org/10.1002/jmr.2326

[11] Wickström, M., Larsson, R., Nygren, P. and Gullbo, J. (2011) Aminopeptidase N (CD13) as a Target for Cancer Chemotherapy. Cancer Science, 102, 501-508. https://doi.org/10.1111/j.1349-7006.2010.01826.x

[12] Schubert, W. (1990) Multiple Antigen-Mapping Microscopy of Human Tissue. In: Burger, G., Oberholzer, M., Vooijs, G.P., Eds., Excerpta Medica, Elsevier, Amsterdam, 97-98.

[13] Schubert, W. (1992) Antigenic Determinants of T Iymphocyte Alpha/Beta Receptor and Other Leukocyte Surface Proteins as Differential Markers of Skeletal Muscle Regeneration: Detection of Spatially and Timely Restricted Patterns by MAM Microscopy. European Journal of Cell Biology, 58, 395-410.

[14] Dress, A., Lokot, T., Schubert, W. and Serocka, P. (2008) Two Theorems about Similarity Maps. Annals of Combinatorics, 12, 279-290.

https://doi.org/10.1007/s00026-008-0351-4

[15] Goyal, N.A. and Mozaffar, T. (2014) Experimental Trials in Amyotrophic Lateral Sclerosis: A Review of Recently Completed, Ongoing and Planned Trials Using Existing and Novel Drugs. Expert Opinion on Investigational Drugs, 23, 1541-1551. https://doi.org/10.1517/13543784.2014.933807

[16] Schubert, W., Masters, C.L. and Beyreuther, K. (1993) APP + T Lymphocytes Selectively Sorted to Endomysial Tubes in Polymyositis Displace NCAM-Expressing Muscle Fibers. European Journal of Cell Biology, 62, 333-342.

[17] Moisse, K., Mepham, J., Volkening, K., Welch, I., Hill, T. and Strong, M.J. (2009) Cytosolic TDP-43 Expression Following Axotomy Is Associated with Caspase 3 Activation in NFL-/-Mice: Support for a Role for TDP-43 in the Physiological Response to Neuronal Injury. Brain Research, 1296, 176-186.

https://doi.org/10.1016/j.brainres.2009.07.023

[18] Barysenka, A., Dress, A.W. and Schubert, W. (2010) An Information Theoretic Thresholding Method for Detecting Protein Colocalizations in Stacks of Fluorescence Images. Journal of Biotechnology, 149, 127-131. https://doi.org/10.1016/j.jbiotec.2010.01.009

[19] Schubert, W., Gieseler, A., Krusche, A. and Hillert, R. (2009) Toponome Mapping in Prostate Cancer: Detection of 2000 Protein Clusters in a Single Tissue Section and Cell Type Specific Annotation by Using a Three Symbol Code. Journal of Proteome Research, 8, 2696-2707. https://doi.org/10.1021/pr800944f

[20] Schubert, W., Friedenberger, M., Bode, M., Krusche, A. and Hillert, R. (2008) Functional Architecture of the Cell Nucleus: Towards Comprehensive Toponome Reference Maps of Apoptosis. Biochimica et Biophysica Acta, 1783, 2080-2088. https://doi.org/10.1016/j.bbamcr.2008.07.019 
[21] Bode, M., Irmler, M., Friedenberger, M., May, C., Jung, K., Stephan, C., Meyer, H.E., Lach, C., Hillert, R., Krusche, A., Beckers, J., Marcus, K. and Schubert, W. (2008) Interlocking Transcriptomics, Proteomics and Toponomics Technologies for Brain Tissue Analysis in Murine Hippocamapus. Proteomics, 8, 1170-1178. https://doi.org/10.1002/pmic.200700742

[22] Bhattacharya, S., Mathew, G., Ruban, E., Epstein, D.B., Krusche, A., Hillert, R., Schubert, W. and Khan, M. (2010) Toponome Imaging System: In Situ Protein Network Mapping in Normal and Cancerous Colon from the Same Patient Reveals More than Five-Thousand Cancer Specific Protein Clusters and Their Subcellular Annotation by Using a Three Symbol Code. Journal of Proteome Research, 9, 6112-6125. https://doi.org/10.1021/pr100157p

[23] Friedenberger, M., Bode, M., Krusche, A. and Schubert, W. (2007) Fluorescence Detection of Protein Clusters in Individual Cells and Tissue Sections by Using Toponome Imaging System (TIS): Sample Preparation and Measuring Procedures. Nature Protocols, 2, 2285-2294. https://doi.org/10.1038/nprot.2007.320

[24] Hillert, R., Gieseler, A., Krusche, A., Humme, D., Röwert-Huber, H.J., Sterry, W., Walden, P. and Schubert, W. (2016) Large Molecular Systems Landscape Uncovers T Cell Trapping in Human Skin Cancer. Scientific Reports, 6, Article No. 19012. https://doi.org/10.1038/srep19012

\section{Note Added in Proof}

The topic of this paper was presented by the author WS at the Global Biotechnology Congress 2017, July 10 to 13, in Boson, MA, USA, with a talk entitled Tissue worlds 2.0: MUSIC robotics decoding the dark space of diseases, experiences with ALS. The content reported here is part of an international patent PCT/EP2016/071867 protecting this treatment option for ALS patients. The complete threshold based analysis of a toponome code based on ref 13 , is found in Schubert W (2003) Topological proteomics, toponomics, MELK technology. Adv Engin/Biotechnol, 83, 189-209. One important question remains to be answered: what is the origin of ACC? My accumulating epidemiologic and MUSIC robotics observations over 30 or more years suggest that the ACC may have an origin inthe epidermis, where they serve as tissue-resident $\mathrm{T}$ cells, which escape from these locations under the influence of heat (sun, sauna). This so called escape-homing to selective brain structures (the pyramidal tract) may be in keeping with the fact, that both epidermis and CNS are derived ontogenetically from the ectoderm. Further in-depth analyses will be started soon. 